\title{
Design of coiled tubing torque reducing device
}

\author{
Chao Xiong ${ }^{1}$, Zhigang Guan ${ }^{1}$, Zhenxing Jiang ${ }^{1}$, Yuchen $\mathrm{Ye}^{1}$ and Zhongxi Zhu ${ }^{2, *}$ \\ ${ }^{1}$ Engineering Technology Institute of PetroChina Xin Jiang Oil Field Company, 834000, Karamay, Xinjiang, China \\ ${ }^{2}$ Petroleum Engineering College, Yangtze University, 430100, Wuhan, Hubei, China
}

\begin{abstract}
During the operation of coiled tubing drilling, the power motor will produce reactive torque when it works, which will affect the drilling process. A torque reducing device is designed to counteract the torque by injecting drilling fluid. The device is designed as a short section, connected with cardan shaft and drive shaft, the nozzle of which can be automatically adjusted according to the pump pressure. The flow model of the device is established by SolidWorks software, and then leaded in FLUENT software for simulation. The standard k- epsilon model is used to simulate the operating conditions of 5 nozzle diameters and 2 outlet diameters respectively, and the parameters such as pressure drop, torque and flow rate are obtained. The designed torque reducing device can offset about $30 \%$ of the torque generated by the screw drill, and the reactive force of single jet is about $152 \mathrm{~N}$. If the jet direction is perpendicular to the axis, it can produce the drilling pressure about $3.4 \mathrm{kN}$. If the direction of the nozzle is aligned with the axis, the effect of pressurization and torsion reduction can be produced simultaneously.
\end{abstract}

\section{Introduction}

Coiled tubing has the advantages of greater curvature, no joint, no need to change diameter, no need to make a connection, continuous roundtrip, the wellhead can be dynamically sealed during drilling operation, drilling equipment been small in size, and so on. [1] In coiled tubing drilling, the drill bit is driven by a downhole power motor, and achieved direction and drilling by controlling bottom drilling tool face. During the rock breaking process, the bit will produce the reactive torque, which will cause the drilling tool to reverse rotation, resulting in the unstable control of the tool face, affecting the drilling speed and continuity. [2] At present, the more advanced horizontal drilling tool - downhole tractor, also known as downhole crawler, can achieve the effect of torque reduction in actual operation. [3-4] But the tractor has a drawback because of its own design that it cannot achieve continuous operation, and will prolong the construction period and increase the cost. In addition, the tractor has many parts, is easy to damage, and has a short service life. [5-6]

According to a torque removing device which has been developed, it uses planetary wheel drives with inside and outside double drill reverse rotation to achieve the purpose of removing reactive torque. But its structure is complex, the work stability is not high, and the service life is short.

In view of the above problems, a jet type torque reducing device is designed. [7-8] The device can inject drilling fluid from the drilling tool into the annular space. High pressure water jet can produce reverse thrust and act on tools to counteract the reactive torque on the coiled tubing, which is beneficial to smooth drilling. The device is installed at the joint between the cardan shaft and the drive shaft, upper the guide hole. The end of the cardan shaft assembly is lengthened to accommodate the reactive torque device. The device is designed as a short section. The upper end and the lower end are respectively threaded with the universal shaft assembly and the transmission shaft assembly.

\section{Design of coiled tubing torque reducing device}

The structure is shown in Figure 1. Coiled tubing torque reducing device is mainly composed of fixed valve core, movable valve core, spring, bearing, limiting baffle plate, outer cylinder and nozzles.

\subsection{Device structure introduction}

The movable valve core and the fixed valve core are in sleeve shape, and the universal shaft penetrates through the valve core. The outer side of the movable valve core is connected with the outer cylinder in a sliding way, and the inner upper part of it is installed with a bearing between the cardan shaft. The design of bearings is to stabilize the cardan shaft and prevent the end of the cardan shaft from uneven loading. The space between the lower part of fixed valve core and the cardan shaft is provided for the drilling fluid to flow. A plurality of through holes are distributed between the upper end face and the inner side of the movable valve core as a drilling fluid passage. The outer side of the fixed valve core is welded with the outer cylinder, and a spacing is provided between the inner side and the universal shaft is left to

\footnotetext{
* Corresponding author: zhuzhongxi@126.com
} 
be used as the outlet of the drilling fluid. The lower part of the movable valve core is sheathed on the upper part of the fixed valve core, and a closed cavity is formed between the fixed valve core, the movable valve core and the outer cylinder wall. The spring is arranged in the cavity and is connected with the movable valve core and the fixed valve core, so that the movable valve core can slide elastically in the axial direction. Limit baffle plate is installed in the upper end face of movable valve core, to limit the sliding range of the movable valve core.

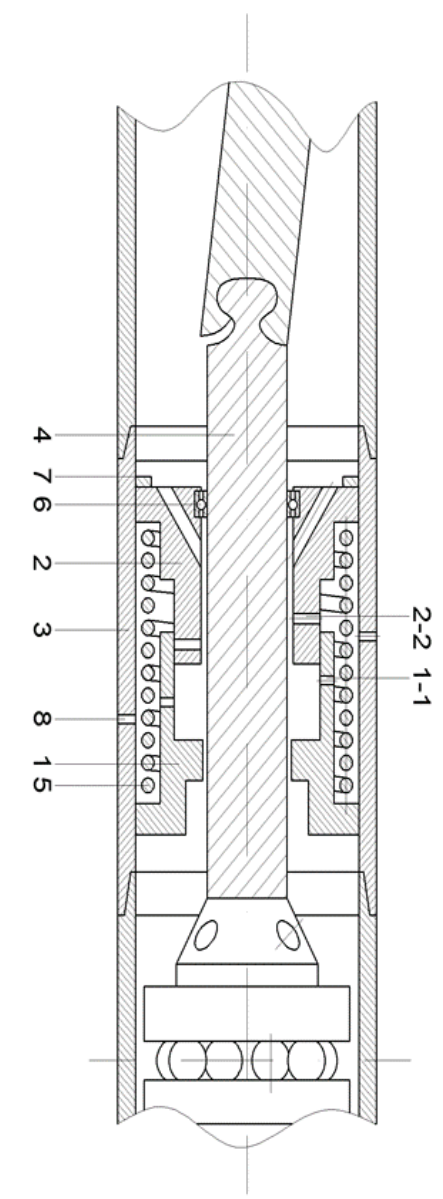

Fig 1 Structural representation map of torque reducing device

1. Fixed valve core, 2. Movable valve core, 3.Outer cylinder, 4. Cardan shaft, 5. Spring, 6. Bearing, 7. Limit baffle plate, 8.Nozzle, 1-1. Through hole, 2-2.

The upper part of the fixed valve core and the lower part of the movable valve core are provided with a plurality of through holes, and the radial direction is distributed in a circle. Its characteristic is the size of the hole being not the same, and the positions of the holes being unevenly distributed on the axial direction. Each of the through holes of the movable valve core is provided with a through hole corresponding to the size and position on the fixed valve core. When the movable valve core moves to different positions, different through holes are connected between the movable valve core and the fixed valve core.

The 24 nozzles are distributed on the outer cylinder. Its characteristic is evenly distributing in the radial direction, and spirally distributing in the axial direction. The nozzle direction is tangential to the outer cylinder in a radial direction. The purpose of the nozzle unevenly distributing is to prevent the nozzle from being in the same plane, so that the strength of the outer cylinder is not enough and easy to be damaged.

\subsection{Work process analysis and design features}

When the high pressure drilling fluid drives the screw drilling tool to rotate, the drilling fluid pressure make spring to be compressed, and the movable valve core moves downward. According to the the pressure of drilling fluid, corresponding numbers of through holes is opened. Then the drilling fluid flows into the cavity and is ejected through 24 nozzles. As the injection direction is tangential to the outer cylinder, the outer cylinder generate torque to counteract the partial torque generated by power motor. When the pump is stopped, the spring is reset, and the movable valve core returns to the limit baffle, and all the through holes are closed and stop working.

The design of the device borrows the principle of the bypass valve. It works when the drilling fluid is circulating, and stops working when the pump is closed. With the change of the drilling fluid flow rate, the flow of jet flow will change. If the direction of the nozzle is adjusted, it can produce drilling pressure while reducing the torque. Due to the effect of spraying, the fluid in the annulus is spiral upward, and make debris more evenly distributed in the drilling fluid.

\section{Reverse torque calculation of device}

Take a nozzle of the device as the object of study to calculate reverse thrust. Jet reverse thrust is the comprehensive effect of pressure and viscous resistance on high pressure cavity and nozzle. Due to the pressure distribution of high pressure cavity is mainly related with the geometrical structure and the size of the nozzle, and it is difficult to measure, so it can be obtained by momentum theorem that jet reverse thrust equal to the rate of the fluid momentum control body[9-11]

For the outlet $\mathrm{E}$ in the direction of the vertical inlet, the acting force on the flow is $\mathrm{F}_{\mathrm{y}}$ :

$$
F_{y}-P_{2} \cdot \frac{\pi}{4} d_{2}^{2}=\rho q v_{2}
$$

Where: $F_{y}$-the nozzle on the flow force, $\mathrm{N} ; P_{2^{-}}$ Outlet pressure, $\mathrm{Pa} ; d_{2}$-Nozzle area, $\mathrm{m} ; \rho$-Drilling fluid density, $\mathrm{kg} / \mathrm{m}^{3} ; q$-Nozzle flow, $\mathrm{m}^{3} / \mathrm{s} ; v_{2}$-Exit velocity, $\mathrm{m} / \mathrm{s}$ 。

The acting force of drilling fluid on nozzle, $\mathrm{R}$ and nozzle acting force on drilling fluid, $F$ is the relationship between reaction force and reaction force.

$$
\mathrm{R}=-\mathrm{F}=-\left(\rho q v_{2}+\mathrm{P}_{2} \cdot \frac{\pi}{4} d_{2}^{2}\right)
$$

Where: R- Reaction of liquid to nozzle.

Because the nozzle area $d_{2}$ is very small, the jet thrust is determined mainly by the jet velocity, in which the jet 
velocity is determined by the inlet flow rate and the nozzle diameter.

\section{Flow simulation of fluid over falling torsion device}

The reduce of the friction and torque device is simulated by FLUENT software, and the force of the device after jet is analyzed.

\subsection{Entity model}

The structural data of a $5 \mathrm{LZ} 89 * 7$ model common screw drill tool is modeled as the original data, The work torque is $560 \mathrm{~N} . \mathrm{m}$, bit nozzle pressure drop is $1.4-7 \mathrm{MPa}$, the WOB is $2 \mathrm{~T}$, the flow range of $3-8 \mathrm{~L} / \mathrm{s}$. Three dimensional geometric structure of fluid flow model is constructed by using SolidWorks software. As shown in figure 1. In order to simplify the model without considering the strength of the device. The hole structure of the entrance of the device is changed into a circular whole, and the spiral distribution of the 24 outlets is arranged in a linear permutation.
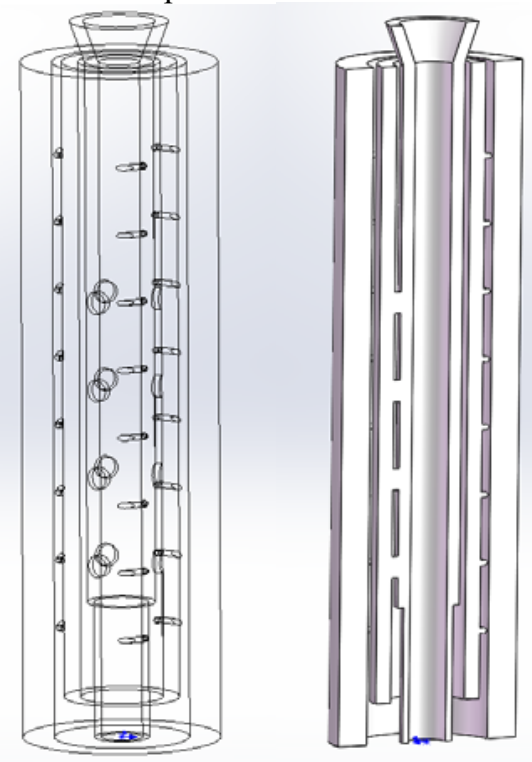

Fig 2 Flow model and half cutaway drawing of coiled tubing reduce of the torque

\subsection{Computational model and boundary conditions}

After the grid components are partitioned in the workbench, the files are read into the Fluent for simulation. The flow velocity of drilling fluid is high, and its flow pattern is viscous turbulent flow, and it is treated as incompressible flow. Therefore, the standard $\mathrm{k}$ - epsilon model is adopted to calculate. In order to facilitate the simulation, choose water as the medium fluid properties, modify the density of $P=1200 \mathrm{~kg} / \mathrm{m}^{3}$, $\mu=0.004 \mathrm{~kg} / \mathrm{m} \mathrm{s}$. Moreover, the density and viscosity are independent of temperature
The boundary conditions and basic assumptions for numerical simulation of fluid flow in a torque reduction device include: The inlet is the velocity inlet, and the inlet flow is set to $8 \mathrm{~L} / \mathrm{s}$; The outlet is the pressure outlet and the boundary condition is pressure $\mathrm{P}=0$; Slip free condition of solid wall; The temperature distribution problem is not considered.

\subsection{Calculation results and analysis}

The nozzle diameters are 3, 4, 5, 6 and 7, respectively, and the lower exit diameters are 40 and 38 respectively, The device is simulated by fluent, and the fluid velocity vector and the velocity distribution of the drilling fluid on the cross section are shown in figure $3 \sim 4$.

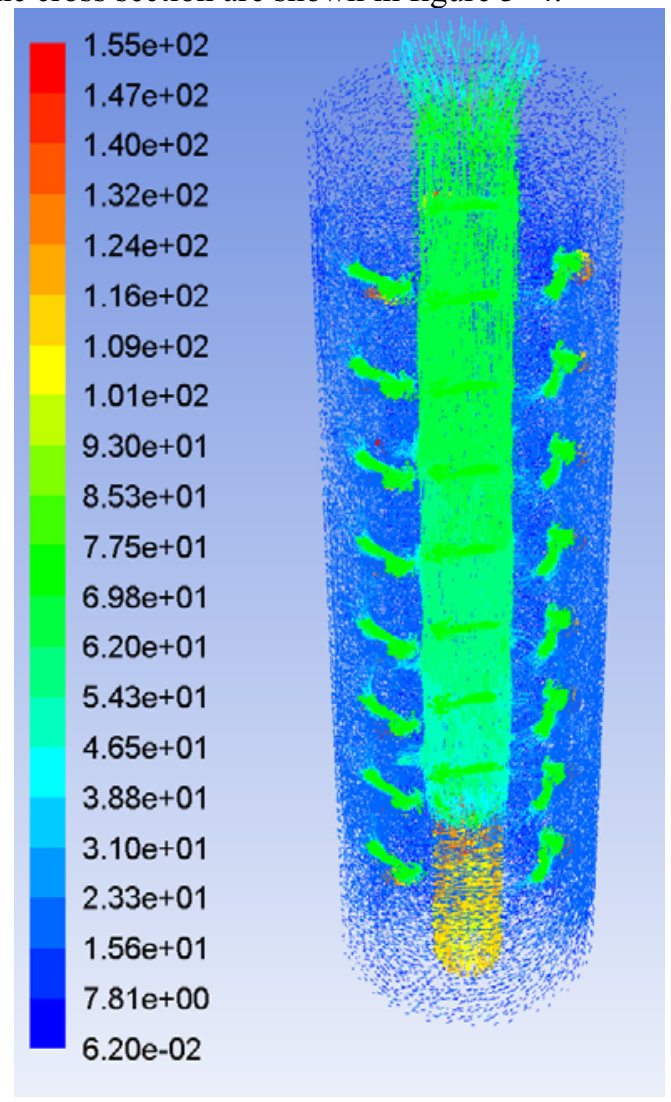

Fig 3 Speed vector diagram of torque reduction device 


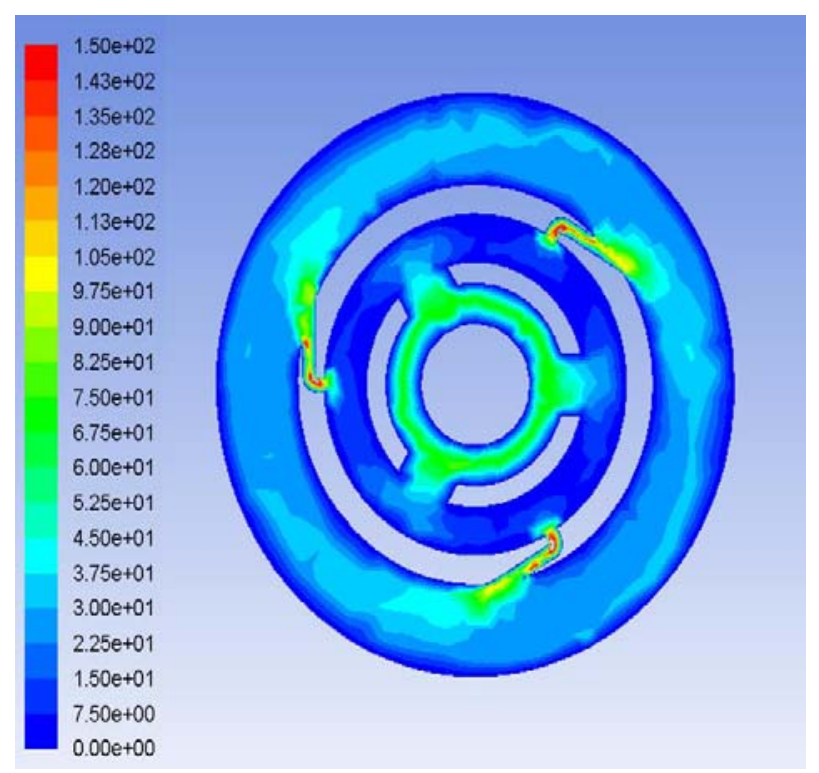

Fig 4 Flow chart of drilling fluid in cross section

It can be seen from the simulation data that the pressure drop decreases with the increase of nozzle diameter. The torque increases with the nozzle diameter, but reaches the maximum when the nozzle diameter is 5 . Reduce the outlet diameter, pressure drop and torque will increase.

Larger torque and smaller pressure drop can be obtained by increasing the nozzle diameter and reducing the lower exit diameter, but the nozzle flow ratio will increase as well. When the nozzle diameter is 7 , the outlet diameter is 38, the nozzle flow accounted for $56.87 \%$, the bit water flow will be too small to affect the chip.

In the simulated data, the maximum torque is 167.9 N.m, approximately $30 \%$ of the rated torque of the screw, and the single nozzle cunterforce is about $152 \mathrm{~N}$. In design, if the nozzle direction is parallel to the wellbore axis, the 24 nozzles can produce up to about 3.4 thrust, which can provide a bit of drilling pressure for the drill bit. According to the design requirement, the nozzle direction and the axis can be made into a certain angle, which can not only reduce the torsion but also increase the drilling pressure.

As the device only tests the drop torque effect through simplified 3D model numerical simulation, the device needs to be optimized and checked to achieve higher performance requirements for the actual downhole environment.

\section{5 conclusions}

(1) A coiled tubing torque reduction device is designed to compensate for the torque generated by a part of the screw drill through the injection of drilling fluid, and the injection quantity can be adjusted according to the pump pressure.

(2) Through calculation analysis and 3D simulation of fluent, the coiled tubing torque reduction device produces about 167.9 N.m torque, which can offset about $30 \%$ of the torque generated by the screw drill.
(3) When the nozzle direction is perpendicular to the axis, the device will produce torque, such that if the nozzle direction is parallel to the axis, an axial thrust of about $3.4 \mathrm{kN}$ can be generated to increase the drilling pressure. When the direction of the nozzle is in a certain angle with the axis, the effect of pressurization and torsion reduction can be generated simultaneously

\section{References}

1. Su Xin-liang, LI Gen-sheng, SHEN Zhonghou, YUE Chun-hong.Progress in coiled tubing drilling Technology research and Application. Natural Gas Industry,2008,(08):55-57+66+140.

2. Zhang Hongwei.Mechanical analysis of coiled tubing. China University of Petroleum,2010.

3. Yang Gang. Research on the influence of coiled tubing drilling torque on buckling and friction. Southwest Petroleum College,1999.

4. Liu Yan, Gu Jiangrui, Wang Junliang.Innovation of coiled tubing traction technology. Foreign Oil Field Engineering, 2009, (03):31-33+54.

5. Wang Xuming, XIA Hongnan, Wang Can.Technology for reducing drag and increasing speed of coiled tubing Drilling. Liaoning Chemical Industry,2014,(09):1165-1167.

6. Ma Zhiqi, ZHANG Xiliang, Shi Hongjuan, Zhang Fenghui.Technical Status and Development Trend of downhole retractors. Petroleum Pipes and Instruments,2015,(04):8-10.Shen Zhonghou Li Gensheng Wang Ruihe. Application and Prospects of Water Jet Technology in Petroleum Engineering. Engineering Science, 2002.

7. Zhang Jun, LI Xiaohui, ZHU Yuquan. Research on the reverse thrust of conical nozzle water jet. Machine tool and hydraulics,2007, (04):139$141+144$.

8. Labus T J, Association W J T. Fluid jet technology : fundamentals and applications. Water Jet Technology Association, 1995.

9. Korde U A. Study of a jet-propulsion method for an underwater vehicle. Ocean Engineering, 2004, 31(10):1205-1218.

10. Daugherty, RobertL. Fluid Mechanics With Engineering Applications . McGraw-Hill, 1977.

11. Hong Y U, Tingkan L U. Numerical Simulation Study on Structure Design and Parameters Optimization of High Pressure Water Jet Cutting Nozzle. Machine Tool \& Hydraulics, 2009. 